\title{
LA CEPAL Y LA SOCIOLOGÍA DEL DESARROLLO ${ }^{1}$
}

Las propuestas de la Comisión Económica para América Latina (CEPAL) se ubican principalmente en el terreno de la economía. Sin embargo, como corresponde a un enfoque integrado del desarrollo, ellas también incluyen aspectos sociológicos y políticos. Los aspectos sociales del desarrollo fueron considerados por la CEPAL desde sus orígenes, y también en estas materias se buscó evitar la traslación mecánica a la región de las teorías existentes. En diálogo con especialistas de otras instituciones se buscó caracterizar la especificidad de los problemas regionales y precisar las condiciones sociales y políticas que facilitarían el desarrollo económico. Se investigó el papel del Estado en la estrategia de desarrollo, así como los potenciales aportes al proceso de desarrollo de los agentes privados y los diversos grupos sociales. También se examinó, en el marco del proceso de integración, el tema de la identidad sociocultural de la región. La combinación de la voluntad política y las propuestas para el desarrollo fue examinada detenidamente en las experiencias de planificación. Las investigaciones han seguido de cerca las modificaciones que la sociedad de la región ha ido experimentando. La evolución del sistema educativo, así como la marginalidad y la pobreza, han si-

1 Texto extraído de la Revista de la CEPAL, N ${ }^{\circ}$ 58, abril de 1996, pp. 191-204. 
do también temas de importancia, al igual que la situación de las mujeres y los jóvenes. En un período en que se perfilan nuevas modalidades de desarrollo, la CEPAL ha analizado el costo social del ajuste, el papel de la educación y las condiciones de participación ciudadana, entre las nuevas condiciones del desarrollo social, siendo este último un componente central de la propuesta de transformación productiva con equidad.

\section{LOS ASPECTOS TEÓRICOS}

Los problemas del desarrollo, tanto económico como social, e incluso político y cultural, tienen una larga data en la reflexión de las distintas disciplinas que a tales temas se vinculan, reflexión que se liga a preocupaciones estrictamente teóricas y también a afanes prácticos de política gubernamental. No obstante, puede señalarse que con posterioridad a la Segunda Guerra Mundial, el tema del desarrollo adquirió un singular relieve; a su promoción no fueron ajenos los distintos organismos de las Naciones Unidas, y el propósito del desarrollo se convirtió en un proyecto internacional. Por cierto, la CEPAL, cuyo ámbito de competencia eran los denominados países latinoamericanos, participó activamente en esa tarea y logró hacerlo con gran originalidad. En lo que se refiere a los "aspectos sociales del desarrollo", los problemas de población, de condiciones de vida, salud, vivienda y educación encontraron cabida entre los temas más estrictamente económicos, y el carácter de la estructura social de los países de la región fue desde temprano - en los primeros años de la década de 1950 — objeto de atención.

Pero hay un hecho que conviene destacar desde el principio: los problemas del desarrollo que se trataba de enfrentar eran a la vez un desafío para la teoría misma de las distintas disciplinas en juego, y aquí es de reconocer el valor que adquirió la llamada "heterodoxia teórica" de la CEPAL. En sus planteamientos y análisis económicos se trataba de utilizar ideas producidas por diferentes escuelas de pensamiento en forma enriquecedora y novedosa, y lo mismo se hizo con los diversos enfoques sociológicos existentes. 
Se consideraba que el tema del desarrollo era en esos momentos motivo de interés no sólo de los científicos o de los políticos, sino también del público en general, y que esta misma condición hacía que los problemas teóricos dejaran de ser disquisiciones de capillas o de escuelas para constituirse en un verdadero estímulo al avance de las ciencias sociales y de la sociología en particular.

Es por eso que de un modo u otro las diferentes orientaciones de la sociología estuvieron presentes, no tan sólo en distintos momentos del análisis cepalino, sino que, además, tendieron muchas veces a combinarse, de tal modo que en algunos de los estudios es posible percibir una perspectiva que es más bien propia de lo que se denomina "sociología crítica", en donde el carácter de la sociedad en que se vive es enjuiciado, apoyándose para tal objeto en una concepción del mundo o ideología. La intención de esta sociología crítica es proponer una transformación de la sociedad concordante con los principios que le sirven de sustento. Si estas orientaciones valorativas han estado presentes - y no hay para qué esconderlo, son las que a menudo han suscitado más críticas-, han coexistido con ellas los propósitos de análisis sociológico de un carácter más funcional, encaminado a dar orientaciones precisas para la conducta práctica. No se pretende en este caso que el análisis sociológico conduzca necesariamente a la reforma o a la transformación completa de la estructura social, sino a actuar de modo eficaz sobre algunos de los componentes de ella, asumiendo metas que, de algún modo, han sido previamente determinadas.

Pero quizás lo que ha predominado es una sociología que intenta comprender una determinada situación social de carácter histórico. En el caso de América Latina se ha intentado mostrar las peculiaridades de su estructura y las tendencias dinámicas de su particular situación, tratando de poner en claro las diversas posibilidades que ellas encierran. En esa perspectiva predomina la intención de diagnóstico y, no eludiendo señalar los resultados previsibles de la elección entre una u otra alternativa, se está consciente de que dicha elección está influida por distintos puntos de vista y pertenece por último a quien corresponde la responsabilidad de la acción. 
Estas tres grandes posturas a que se ha hecho alusión, y que de algún modo, incluso entremezclándose, han coexistido en los análisis sociológicos de la CEPAL, llevan también a definir el papel del sociólogo en las tareas del desarrollo, que conviene no olvidar, eran, por lo menos en los inicios, preferentemente definidas como de desarrollo económico. Es así que los sociólogos de la institución consideraban que sus funciones oscilaban entre las de simple colaboración en las labores más esenciales del economista (determinar y definir un campo específico de investigación y elaboración teórica) o en el cumplimiento de su papel crítico, de participación en la elaboración de las orientaciones generales —incluso políticas - del desarrollo económico.

Si bien los sociólogos no carecían de teorías que les permitieran orientarse en el conocimiento de la realidad que enfrentaban, la mayor parte de las hipótesis derivadas de ellas, que por lo general eran admitidas como válidas, provenían de la abstracción de una experiencia histórica correspondiente a medios sociales distintos al latinoamericano. La pretensión de actuar de manera racional sobre la realidad se fundaba en el hecho de disponer de un conocimiento científico apropiado; pero el problema era saber hasta qué punto tal conocimiento era adecuado o inadecuado para América Latina, dado que las teorías habían sido en lo fundamental concebidas en Europa o en los Estados Unidos. Se imponía por consiguiente un trabajo de investigación empírica, que además de hacer aportes concretos al conocimiento, permitiría confirmar o desechar hipótesis en boga y, por qué no, proponer algunas nuevas. En suma, la sociología compartía la orientación que era distintiva del pensamiento económico de la CEPAL: la no traslación mecánica de las teorías existentes.

Como lo advirtieran algunos autores, las ciencias sociales, en su intención de promover a través de la temática del desarrollo una práctica social racional, cumplieron un papel singular. Si se asume como válida la interpretación de Max Weber sobre el carácter del Occidente moderno, donde el conjunto de las relaciones sociales ha sido permeado por el racionalismo, la ciencia habría encontrado en este proceso general de racionalización su 
lugar adecuado; habría, por consiguiente, una afinidad entre la práctica científica y la racionalidad de la práctica social. En América Latina la racionalidad era, por lo menos en parte, introducida por la ciencia. Se pretendía que la respuesta al desafío del desarrollo se encontraba en gran medida en la ciencia misma, a la que se concebía como un valor; la crítica de la realidad señalaba que ésta no se conformaba a los postulados de la razón. La ciencia, sin plena conciencia quizás, se convertía en ideología.

En cierto modo, el contraste entre teoría y realidad llevó a un intento de determinar la especificidad de América Latina. Varias hipótesis que las distintas teorías del desarrollo postulaban como válidas no encontraban confirmación en estos lugares; por ejemplo, la de una correspondencia lineal entre urbanización e industrialización, la existencia de una rígida dualidad tradicionalmoderna, o la existencia de una burguesía industrial que asumiera como propio un proyecto de desarrollo nacional. Estas primeras verificaciones obtenidas por contraste con la teoría permitían señalar lo que América Latina no era, pero aún era difícil señalar lo que sí era en propiedad.

Por cierto que no fue sólo la sociología de la CEPAL la que se propuso como tarea la búsqueda de la especificidad de América Latina: ésta fue labor de la casi mayoría de los sociólogos latinoamericanos, y justo es reconocerlo, habían sido ya precedidos por historiadores, pensadores, literatos y otros estudiosos; no obstante, el tema del desarrollo hacía más urgentes las respuestas. Era necesario determinar la particularidad del comportamiento de los distintos grupos sociales (la burguesía, los sectores medios, las clases populares), del problema de la marginalidad y de la posición social del indígena o del negro, y además comprender cuál era el rasgo específico de sus instituciones sociales, del Estado, las fuerzas armadas y así por delante.

En la búsqueda de la adecuación de la teoría sociológica a los problemas del desarrollo económico latinoamericano no puede dejar de mencionarse, tanto por su labor pionera como por su influjo permanente, la obra que llevó a cabo en la CEPAL don José Medina Echavarría. Su referente teórico fue Max Weber, quien, 
al establecer una distinción entre instituciones (o comunidades) económicas que se caracterizan por la búsqueda de un beneficio o por la cobertura de necesidades, y las instituciones (o comunidades) "económicamente relevantes", en donde las actividades que llevan a cabo no son económicas pero pueden representar un "momento causal" en el proceso de una actividad económica, permitió establecer el nexo entre el análisis estrictamente económico y el análisis sociológico. El problema consistía en determinar el grado de adecuación o inadecuación —afinidad electiva, señalaba Weber- entre estos diversos tipos de actividades. La distinción weberiana había sido de hecho también recogida por la teoría sociológica del desarrollo, en esos momentos en boga, que diferenciaba entre los "requisitos de primer orden", que correspondían a las instituciones económicas propiamente tales, como la propiedad, la mano de obra y las relaciones de mercado, y los "requisitos de segundo orden" (instituciones económicamente relevantes), particularmente el régimen político, la ciencia y la técnica, así como las formas de la estratificación.

Aceptando la formulación weberiana que permite distinguir entre lo económico y lo sociológico, pero busca establecer sus conexiones, se planteaban, sin embargo, importantes desafíos teóricos. El análisis hecho por Weber del desarrollo capitalista para un momento histórico determinado, e incluso para un espacio cultural concreto, se había constituido en un paradigma de la forma del desarrollo, y era un hecho que la situación histórica había variado y que el desarrollo del capitalismo tenía lugar en condiciones diversas a las que habían dado origen al modelo teórico weberiano; por otra parte, ya existía con plena vigencia el "modelo soviético", cuyos supuestos o fundamentos eran distintos, tanto en las orientaciones de racionalidad de quienes eran sus agentes como en las condiciones sociales y políticas que condicionaban su organización.

Desde un punto de vista teórico, el desafío que se enfrentaba era de gran magnitud: se trataba nada menos que de construir un nuevo "modelo", que diera cuenta de las condiciones sociales que harían posible el desarrollo económico en las actuales circuns- 
tancias. Entre las nuevas condiciones del desarrollo capitalista se destacaban la no existencia de una competencia perfecta y, por consiguiente, la inexistencia de una plena libertad de mercado; los cambios en las relaciones de trabajo, en las cuales el libre contrato tendía a ser reemplazado por la capacidad de acción y negociación de las organizaciones colectivas, con cierta tendencia de ésta a controlar los puestos de trabajo; y un importante cambio en las funciones del Estado, que dejaba de ser neutral e intervenía regulando la actividad económica o participando directamente en ella.

Estos cambios no sólo tenían vigencia en los "países centrales", sino que ya se manifestaban en muchos de los países latinoamericanos, o por lo menos constituían parte del nuevo modelo al que se aspiraba. De particular importancia para América Latina era la confirmación de la profunda transformación social que estaba teniendo lugar, que se manifestaba en la presencia de nuevas capas sociales movidas por un fuerte impulso de ascenso; tal rasgo era particularmente visible en las clases medias y en el proletariado organizado. Nuevos grupos demandaban condiciones políticas que hicieran posible su plena incorporación a la vida nacional, y a menudo se producían choques de esas capas con las que ya tenían una posición establecida.

Dadas las nuevas condiciones, ¿cómo influirían ellas en la conformación de las motivaciones de conducta que se postulaban como necesarias para el logro de un desarrollo económico sostenido? En términos concretos, ¿cómo era posible el desarrollo de hábitos de trabajo propios de la actividad empresarial o de los correspondientes a la mano de obra? La vida económica moderna supone la existencia de rasgos sicológicos que implican actitudes racionales y cierta disciplina. ¿En qué condiciones se formarían ahora esos rasgos? La "racionalidad económica", ¿con qué otros procesos de racionalidad se relaciona? En la experiencia europea, el moderno capitalismo racional había encontrado condiciones sociales de despliegue en la medida en que había coincidido con cierto tipo de racionalidad del derecho y de la administración, cuyo origen no estaba en motivos estrictamente económicos, sino 
que éstos habían sido de índole política, profesional o ideológica. El tipo de racionalidad, por la cual el derecho y la burocracia se orientaban, era lo que se conoce como "racionalidad formal" —la que se atiene estrictamente a la norma o al reglamento-- pero era ésta la que hacía posible el cálculo que toda actividad económica requiere. Precisamente en este ámbito se había producido un gran cambio: el Estado, al dejar de ser "neutral”, orientaba tanto el derecho como la burocracia en términos de una "racionalidad funcional" —-tendiente a conseguir el logro de fines y metas-, en desmedro de la pura racionalidad formal.

Varios problemas se presentaban en el caso de América Latina respecto de las conductas económicas de la población, si se deseaba que éstas fueran adecuadas al objetivo de desarrollo económico que se perseguía. Es propio de una intención colectiva de desarrollo el que se aspire a un mejor nivel de vida o de consumo, pero también se requiere cierto sentido de responsabilidad, tanto individual como colectiva. Ambas disposiciones no necesariamente coinciden; incluso pueden ser contradictorias: los deseos de un mejor nivel de vida pueden no estar acompañados del impulso a realizar el esfuerzo necesario para conseguirlo; la aspiración individual al goce puede no estar contenida por el sentido de una responsabilidad colectiva. De modo que, por no estar asegurada la necesaria concordancia entre una y otra disposición, aparecía como necesario promoverla conscientemente, papel que podía ser competencia de instituciones no estrictamente económicas, como el sistema educativo, los medios de comunicación pública, el Estado, los partidos, los sindicatos y muchos otros. Pero se señalaba como un elemento quizás clave el que los grupos dirigentes proporcionaran un modelo de conducta que pudiera ser imitado, ya que la desorientación o la atonía de las masas se debía muchas veces a la ausencia de conductas ejemplares de las elites dirigentes, tanto económicas como políticas.

De igual forma, en los planteamientos iniciales de la "sociología del desarrollo" de la CEPAL, existió conciencia de los problemas de la relación entre política y desarrollo económico, aunque por cierto el tema no fue sólo preocupación de la "sociología cepali- 
na”. En América Latina, muchas de sus formas políticas parecían incompatibles con la racionalidad económica requerida; gran parte de los partidos o de los gobiernos carecían de ideologías racionalizadas y de aparatos políticos que tuviesen ese carácter; podía darse en ellos un interés por el crecimiento económico, pero esto a menudo no obedecía necesariamente a impulsos racionales. Pero además de este hecho es necesario reconocer también que tanto la racionalidad política como la racionalidad económica tienen finalidades que les son propias, y en un momento en que el Estado actúa, por su capacidad de regulación o de modo directo, en el ámbito de la economía, es necesario establecer conexiones entre los tipos de racionalidad —económica y política—y admitir que los compromisos que pueden lograrse entre ambas sólo adquieren el carácter de un compromiso temporal. El problema planteado respecto a la relación entre racionalidad política y racionalidad económica se hizo mucho más visible cuando la CEPAL abordó el tema de la planificación.

Otro elemento que caracterizaba las condiciones sociales en que el desarrollo económico podía tener lugar en América Latina, y que por lo tanto debía ser motivo de reflexión, era el del casi inevitable conflicto político-social. El propio desarrollo - $-\mathrm{y}$ sobre todo en los momentos en que adquiría un ritmo mayorimplicaba desajustes de la estructura social, creando situaciones de conflicto. Era posible prever que se produjera un desarraigo acelerado de un importante número de personas, muchas de ellas de origen campesino, que no podían adaptarse con facilidad a las nuevas condiciones, precisamente por la misma rapidez con que el cambio tenía lugar. También era previsible que se originaran fuertes desigualdades - motivo de conflictos - entre distintos sectores de la vida nacional, desniveles graves entre regiones o diferenciaciones de grados de modernización y desarrollo en el interior de las distintas actividades económicas. Tampoco dejaba de preocupar la expansión de aspiraciones propias de las "sociedades de consumo”, cuyo modelo eran los países más avanzados, en condiciones difíciles de ser satisfechas. En suma, había que 
tener en cuenta que el conflicto económico-social era un componente casi inevitable del propio desarrollo económico.

Es de destacar la importancia que se otorgaba al sistema político, puesto que el desarrollo se concebía como el resultado de un programa formulado con claridad y que requería para su cabal funcionamiento de un marco político apropiado; de tal modo que muchas veces se pensó que las diferencias entre los países latinoamericanos no obedecían tanto a aspectos económicos, sino a la infraestructura social y a los procesos políticos que ésta permitía. En esto había plena coincidencia con la propuesta global que desde sus inicios la CEPAL planteaba. El Estado no solamente debía intervenir en el proceso de desarrollo, mediante políticas de protección de las industrias nacientes y el fomento a la modernización del sector primario exportador, sino que se le concebía como un promotor de procesos y no sólo como corrector de fallas o ineficiencias; existía coincidencia con las propuestas de Gunnar Myrdal en este campo, para quien las funciones de la intervención estatal en los países industrializados modernos debían ser sobre todo de arbitraje y de nivelación, y en los países menos desarrollados, antes que nada, de orientación y programación. Por lo demás, la propia situación histórica obligaba al Estado a asumir nuevas funciones o a desempeñar un papel más activo que el tradicional; era necesario hacer frente a problemas de la coyuntura internacional cada vez más graves y complicados; los procesos de transformación internos generaban tensiones y conflictos, y además había que llevar a cabo una urgente política social, dado que la estructura tradicional de la sociedad se concentraba en un proceso de profunda modificación y reajuste.

Por cierto, no todo se reducía a la capacidad de acción del Estado; era preciso promover cambios en las orientaciones de conducta de algunos grupos sociales claves para el logro del desarrollo económico. Se tenía conciencia, por ejemplo, de que en América Latina predominaba el "empresario comercial" sobre el "empresario industrial”, que a menudo el empresario actuaba más en términos de coyuntura que en términos de programación de mediano y largo plazo, o que trataba de funcionar escudado en 
el amparo de determinadas alternativas de poder. Se trataba entonces de generar nuevas actitudes del empresario, tanto público como privado, en su carácter de gestor e innovador económico. De igual modo, con relación a la mano de obra, se señalaba la necesidad de constituir un ethos del trabajo, capacidad de adaptación técnica y conciencia de responsabilidad social estrechamente asociada a la conciencia de su propia función en el conjunto de la sociedad. Esto sólo podía lograrse con un aumento de la escasa participación que estos grupos tenían en el ámbito nacional, en donde a menudo se obstaculizaba el adecuado funcionamiento de las organizaciones obreras.

No debe dejar de mencionarse que en este esfuerzo por trazar una perspectiva de desarrollo para la región, la CEPAL encaró desde el principio el tema de la integración. Ésta aparecía como una necesidad vinculada al proceso de industrialización, pues se requería el establecimiento de mercados que permitieran economías de escala y además una unificación de esfuerzos en los procesos de modernización. El propósito de integración, sin embargo, exigía un mejor conocimiento de la identidad sociocultural de la región y planteaba desafíos muy concretos. El hecho es que en América Latina el proceso de integración nacional en la mayor parte de los países ha sido muy lento y podría afirmarse que en la mayoría de los casos sólo se produjo en el siglo XX. La interrogante era: ¿cómo se produce la integración supranacional de nacionalidades recién nacidas?

El objetivo de desarrollo que la CEPAL trataba de formular implicaba, con originalidad, una aspiración de modernidad, pero se postulaba que esos propósitos podían ser perseguidos deliberadamente tanto en el plano económico como en el social, que se podían prever las tensiones y actuar sobre ellas, y que esta capacidad de acción podía transformarse en una dinámica endógena de las sociedades en cuestión. Así como se proponían "reformas estructurales" en el ámbito de la economía, se aconsejaban también reformas estructurales en el social; por ejemplo, se propiciaba una reforma educativa que promoviera la movilidad social y permitiera internalizar nuevos valores y modos de conducta, una refor- 
ma agraria que modificara la tenencia de la tierra para dar paso a nuevas formas de relación social, una reforma del Estado y del sistema político para adecuarlo a las necesidades de un desarrollo consciente y planificado. Como temas constantes de la reflexión aparecían el de la conexión entre desarrollo económico y desarrollo social, la autonomía nacional y la democratización política.

\section{LOS PROBLEMAS DE LA PRAXIS}

El esfuerzo de elaboración teórica que llevaba a cabo la CEPAL requería, por cierto, una confirmación práctica y que sus postulados se mostraran conformes a la realidad. Aproximadamente desde mediados de los años sesenta, el proceso de desarrollo mostraba fuertes dificultades en algunos países significativos de la región, e incluso algunas tendencias al estancamiento de su dinámica; el supuesto de que la nación fuera el ámbito donde se procesaran las decisiones políticas que más influían en la orientación del desarrollo, se veía en entredicho por la importancia que adquirían los centros externos de decisión: incluso los sistemas políticos institucionales no aparecían como permeables a una mayor participación. Las premisas de la opción de desarrollo que se postulaba aparecían en la práctica como de gran fragilidad.

La CEPAL, en el ámbito económico, había subrayado con singular énfasis los efectos negativos que para los países de la región tenía el tipo de relación entre países centrales y países periféricos, la cual llevaba a una división internacional del trabajo que dificultaba las opciones de desarrollo latinoamericano. Los análisis sociológicos que se emprendieron trataron de mostrar que no era suficiente analizar el tema sólo en términos de "obstáculos externos" al desarrollo, noción en la que permanecía la idea de que el problema derivaba de las relaciones entre sociedades fuertes y otras más débiles a causa de su desigual poder económico; sin embargo, el problema era más complejo, puesto que podía mostrarse que en casos en que había tenido lugar una expansión de la economía industrial, ello había conducido a un entrelazamiento creciente de intereses locales con intereses monopolistas extran- 
jeros. Por lo demás, la presencia de intereses políticos externos se hacía cada vez más manifiesta en la estructura del Estado.

El "problema de la dependencia" — título más difundido para el tratamiento de estos temas- llevó a analizar las condiciones de existencia y funcionamiento del sistema económico y del sistema político, lo cual puso de manifiesto qué tipo de vinculaciones existía entre ambos, tanto en lo que hace al plano interno de cada país como al plano externo. El supuesto principal era que el modo de integración de las economías nacionales al mercado internacional suponía formas de interrelación de los grupos sociales de cada país entre ellos y con los grupos externos. La dependencia no era simple explotación y coacción: existía comunidad de intereses entre grupos dominantes locales y externos; la dependencia no enfrentaba al "conjunto" de los intereses de una sociedad con otra. La interdependencia era un hecho, y eso vinculaba sociedades desarrolladas con sociedades subdesarrolladas, pero también permitía establecer correspondencia de intereses entre los grupos dominantes de ambas sociedades. Si desde esta perspectiva se planteaba una opción de cambio, su dinámica debía radicar en la transformación de la estructura de clases, sectores y grupos sociales, y en las modificaciones del sistema de dominación, lo que demandaba una acción colectiva y una decidida voluntad política.

Quizás donde mejor se pueden observar las dificultades para aunar la voluntad política con la propuesta de desarrollo es en la experiencia de planificación latinoamericana, con la cual la CEPAL y el Instituto Latinoamericano y del Caribe de Planificación Económica y Social (ILPES) se comprometieron fuertemente. Como los mismos autores cepalinos señalaban, la planificación en América Latina tuvo lugar a partir de la pérdida de dinamismo del comercio exterior como impulsor de la actividad económica; esto llevó a reconocer la necesidad de intervención gubernamental para conducir el proceso económico y aceptar ciertos tipos de políticas que corrigieran los efectos de esa pérdida. De hecho, se trataba de promover el cambio hacia un nuevo patrón de desarrollo por agotamiento del anterior. Sin embargo, el poder político —al que correspondía poner en marcha el proceso- mostraba 
cierta ambivalencia: se reconocía que podía ser un instrumento valioso y existían antecedentes inmediatos en Europa occidental que lo justificaban, pero a la vez se le temía por las intenciones de transformación y cambio que contenía.

En lo que respecta de modo más directo a la CEPAL, las propias Naciones Unidas auspiciaban la idea de planificación del desarrollo y se suponía que sus orientaciones valóricas estaban ya resueltas a través de la Declaración Universal de los Derechos del Hombre, la Estrategia Internacional de Desarrollo y otras resoluciones que habían logrado el apoyo de casi todos los países representados. En las formulaciones de las Naciones Unidas se hacía especial hincapié en el desarrollo social; se pretendía que las políticas colocaran en pie de igualdad los aspectos sociales y los económicos, y que se procurara desarrollar una metodología de planificación social como contrapartida de la planificación económica, intentando alcanzar así un enfoque unificado para el análisis y la planificación del desarrollo; de este modo se daría a las necesidades humanas una mejor respuesta que las obtenidas hasta ese momento. Tales propósitos se basaban en la existencia de un consenso sobre valores de bienestar humano y justicia social. Aceptados tales supuestos, el problema era: ¿a quién dirigir las recomendaciones y dar asesoramiento?

Existían expectativas de que la propia sociedad, en concreto los más directamente interesados, se movilizara para el logro de sus objetivos, para lo cual era preciso que se abrieran canales de participación. No obstante, las esperanzas mayores estaban puestas en la capacidad de acción y en el compromiso de quienes cumplían funciones políticas directas. De hecho, en muchos países latinoamericanos se formularon planes con objetivos que implicaban medidas drásticas en temas como la propiedad de la tierra agrícola, y reformas monetarias y crediticias o reestructuración del sistema impositivo con miras a financiar políticas sociales o de otro orden; la mayor parte de las veces estas medidas no pudieron llevarse a cabo porque los gobiernos se vieron sometidos a fuertes presiones (de los perjudicados); en algunos casos, los propósitos que se formulaban correspondían más al 
ánimo de los planificadores que a las convicciones de los políticos y de los ejecutivos de los organismos públicos. Por otra parte, la sensibilidad de los políticos se dirigía mucho más a los síntomas de las situaciones y procuraba tener respuestas inmediatas a ello, en tanto que el planificador tendía a presentar un cuadro de soluciones cuyo fundamento era una concepción estructural del desarrollo que, por consiguiente, se proyectaba más en términos de mediano y largo plazo.

Las dificultades para articular las demandas de corto plazo y las propuestas de política de mediano y largo plazo se agravaban por la inestabilidad política de la mayoría de los gobiernos de la región; los frecuentes cambios de los encargados de la dirección de la cosa pública y el consiguiente cambio de orientaciones hacía que el mediano y largo plazo casi no tuviesen sentido. Además, a menudo la base de apoyo político tendía también a ser inestable, y los gobiernos destinaban su capacidad de maniobra a obtener el apoyo de los principales grupos de presión, por lo cual era frecuente que se utilizaran los instrumentos de política - económica y social — como soportes para lograr un respaldo que permitiera un mínimo de estabilidad. Por último, el compromiso con los fines y medios de la planificación adquiría rasgos de labilidad, porque se pensaba que atenerse de manera más o menos estricta a lo programado restringía la capacidad de decisión del poder político.

Respecto a la capacidad de la propia sociedad para movilizarse en pos de objetivos de desarrollo económico y social, se tropezaba también con dificultades. Los grupos que contaban con una mayor cuota de poder económico y social tendían a identificarse con la situación tal y como existía, y desplegaban estrategias de grupos de presión para influir sobre el sistema de decisiones y defender las posiciones alcanzadas y, en muchos casos, incluso tratar de obtener nuevos privilegios. Los de situación menos privilegiada recurrían, cuando les era posible, a manifestar su disconformidad a través de varias formas de protesta, pero a menudo no estaban en condiciones de actuar coherentemente, y la dependencia de hecho frente al Estado era un rasgo más fuerte de lo que se supo- 
nía. Las expectativas de "participación popular" con capacidad de creación, que se consideraban esenciales ante un objetivo de desarrollo que cumpliera con los fines de bienestar humano y justicia social, no lograban cumplirse, ya que la participación era escasa y efímera.

La práctica mostraba que los procesos reales de la economía y las relaciones sociales que se constituían distaban mucho de elevar apreciablemente el bienestar humano. Los intentos de concebir y aplicar estilos de desarrollo alternativos, con énfasis en la autonomía y la originalidad y orientados a las personas, terminaban pareciendo formulaciones utópicas si se confrontaban con la realidad; cierto grado de frustración era casi inevitable. Incluso en algunos momentos se puso en entredicho la compatibilidad del "desarrollo" —casi siempre referido a sus aspectos más inmediatamente económicos_y la participación; aún más, se dudó de su compatibilidad con las instituciones y los procesos democráticos. Las soluciones tecnocráticas y a menudo autoritarias parecían ser la nueva panacea del desarrollo.

En suma, la experiencia de planificación en América Latina tuvo, a no dudar, algunos logros ciertos, y en el campo social no deben olvidarse sus avances reales en la ampliación de la cobertura de servicios como los de educación, salud o vivienda. No menos importantes fueron los diagnósticos que se alcanzaron, que permitieron conocer mejor la realidad y tener mayor conciencia de las repercusiones políticas y sociales de las decisiones económicas; pero por cierto no fue posible colmar las expectativas que se cifraban en la planificación en cuanto instrumento eficaz de acción práctica. Pero para ser justos no todo puede achacarse a los “errores y equívocos de la planificación". La magnitud de los obstáculos — de toda índole- que encontró, no puede dejar de considerarse.

\section{UNA ÉPOCA DE CAMBIOS Y PROCESOS CONTRADICTORIOS}

Una mirada retrospectiva que intente abarcar los años que transcurrieron desde finales de la Segunda Guerra Mundial hasta el 
momento actual no deja de verse perturbada por la complejidad de los procesos de cambio ocurridos; los juicios positivos o negativos no dependen sólo del talante de quien observa: lo positivo y lo negativo se entremezclan en la propia realidad. Por ejemplo, es innegable la expansión de los mercados internos, y en la mayor parte de los países se ha conseguido un significativo desarrollo industrial. No obstante, a pesar de las expectativas inicialmente cifradas en la industrialización, en general ella no ha podido desempeñar el papel de dinamizadora del conjunto de la economía y generar una demanda de empleo productivo lo suficientemente amplia, y sólo en muy pocos casos tiene un peso importante en el conjunto de las exportaciones.

Por lo tanto, la participación de los países latinoamericanos en el comercio internacional, durante la mayor parte del período, siguió basada en los bienes primarios, y su sector externo estuvo constantemente sujeto a la demanda internacional de estos bienes y a la inestabilidad constante de sus precios. En los casos de exportaciones manufactureras, muchas de ellas correspondían a la elaboración de recursos naturales, y sus precios lamentablemente funcionaban a menudo en condiciones similares a las que caracterizaban a los mercados de productos primarios. Se podría seguir señalando una serie de transformaciones de otro tipo, por cierto de gran impacto, no sólo económico sino también -y a veces preferentemente- de carácter social, como el cambio del sector agrario; pero el hecho es que las transformaciones ocurridas que incidieron en las estructuras sociales y dieron origen a importantes procesos de movilidad generaron, no obstante, nuevas desigualdades y, por ejemplo, la diferencia en la distribución del ingreso ha tendido a mantenerse e incluso a agravarse. Si en esta mirada panorámica al período de posguerra se consideran algunos indicadores básicos de condiciones de vida, la mejoría que se observa es notable, pero aun así grandes grupos permanecen en condiciones de pobreza absoluta.

El hecho es que los patrones distributivos reflejan de modo muy claro las características reales del tipo de desarrollo alcanzado. En él es visible una fuerte heterogeneidad estructural, y 
los procesos de modernización — que a menudo significan uso intensivo de capital físico - no logran incorporar la totalidad de la oferta de mano de obra, ya que la estructura de calificaciones de que se dispone no responde a la nueva demanda. El progreso técnico se concentra en algunos sectores y no alcanza a amplios estratos de baja productividad.

Siendo innegables entonces los grandes cambios que se han producido como consecuencia de los procesos de urbanización, de industrialización y de lo que puede llamarse "modernización dependiente", éstos han tenido efectos paradójicos: grandes sectores de la población, y quizás la mayoría en uno o dos de ellos, se identifican plenamente con los estilos de vida y las expectativas de las modernas sociedades de consumo. En la implantación de esas nuevas modalidades de vida y tipos de aspiraciones desempeñan un papel importante las empresas transnacionales y los mensajes estandarizados de los medios de comunicación, pero es un hecho que han sido internalizados por los estratos que están en condición de hacerlo y por muchos que, si no tienen las condiciones, aspiran a conseguirlas. Pero, lamentablemente, no se trata de una inofensiva "manía imitativa"; se ha generado un patrón de "consumo imitativo" que implica altos costos en términos de recursos internos. Hay una expansión continua del consumo, esencialmente gracias a la prosperidad del $30 \%$ o a lo más del $40 \%$ de la población. Estos sectores requieren ingresos cada vez más altos que les permitan pagar los precios del tipo de bienes que desean. Además, la producción de esos bienes demanda una movilización de recursos - capital, tecnología, trabajo e incluso recursos naturales- que en su mayor parte no corresponden a aquellos de los que los países de la región realmente disponen.

No está por demás recordar la frecuencia de ciclos de altas y bajas en el desempeño económico que han contribuido a hacer más compleja aún la confusa trayectoria del desarrollo latinoamericano desde la posguerra; los momentos de cambio no sólo han coincidido con los momentos de auge; fuertes transformaciones estructurales han tenido lugar en momentos de crisis, y hasta es posible que en algunos casos las crisis las hayan provocado. Por 
ejemplo, en los años ochenta hubo profundas transformaciones, pero a finales de 1989 el producto interno bruto medio por habitante en la región fue inferior en $8 \%$ al registrado en 1980, y equivalente al de 1977 . Pero ese deterioro tuvo un sesgo marcadamente regresivo: para amplios sectores de los grupos medios y populares, los años ochenta fueron un enorme retroceso. En términos generales se estimaba que en 1980 unos 112 millones de personas - $35 \%$ de los hogares de la región — vivían por debajo de la línea de pobreza, cifra que en 1986 aumentó a 164 millones (aproximadamente 38\% de los hogares).

Al confuso panorama económico y social a que se ha hecho somera referencia, se agregaban las complejidades en el campo político; en los años posteriores a la Segunda Guerra Mundial, regímenes de distinto signo se habían sucedido o alternado bruscamente, y en los años setenta y gran parte de los ochenta, las esperanzas cifradas en la constitución estable de regímenes democráticos parecían no tener mucha vigencia en el conjunto de la región. Los estudios sociológicos de la CEPAL se orientaron preferentemente a analizar los cambios ocurridos, en especial aquellos vinculados a la profunda transformación de la estructura social. En términos de población, el puro aumento del número ya planteaba problemas de importancia; había cambiado la proporción de la población rural respecto a la población urbana, y la región podía definirse en el presente y en el futuro próximo como esencialmente urbana, lo que significaba que la forma de organización societal predominante había cambiado, constituyéndose en modelo de ella un sistema urbano secular, y por consiguiente, también se habían transformado las relaciones sociales.

Respecto de los cambios en la estructura económica, con evidentes efectos en las relaciones sociales, era notorio que en la casi totalidad de los países se había constituido un "mercado nacional". Pero las formas de constitución de ese mercado presentaban fuertes distorsiones determinadas por la tendencia a la concentración, lo que a su vez significaba disparidades regionales y fenómenos de exclusión y marginalidad. Se observaba también, como se ha dicho, una producción industrial de bienes que empe- 
zaba a articularse con otros sectores de la economía, como el agro y los servicios, modificando los tipos de organización y relación social que les habían sido propios. En materia de empleo, se había incrementado el industrial y también el terciario; en este último era preocupante la magnitud que alcanzaba el sector de servicios de baja productividad, pero no era desdeñable la significación que adquiría el llamado "terciario moderno". La CEPAL advertía que además de los graves problemas de disparidad en la estructura económica y social, la "economía moderna" que empezaba a surgir planteaba nuevas exigencias, tanto en lo que respecta a la calificación y capacitación de la fuerza de trabajo en todos sus niveles como en lo referente a nuevas formas de organización social (sistema de relaciones laborales, papel de los sindicatos, organizaciones intermedias y otras formas de participación).

Como es evidente, la estructura del poder social también se vio afectada por los cambios señalados; existía una indiscutible presencia de masas, y el recurso a la exclusión coercitiva —que, de más está decirlo, muchas veces se puso en práctica- no resolvía el fondo del problema: lo que de hecho estaba en juego eran las formas de participación, representación y, muy concretamente, el papel de los partidos políticos. Los estudios de la CEPAL se dirigieron preferentemente al análisis de los cambios en la estratificación social, dado que éstos podían explicar las nuevas condiciones en que las relaciones de poder y el conjunto de las relaciones sociales tenían lugar, e influían a la vez sobre las modalidades reales y posibles del desarrollo económico-social.

Estos estudios mostraron que la estratificación social se había hecho más compleja y que en la mayoría de los países había aumentado la proporción de personas que ocupaban posiciones "medianas" o "superiores", aunque el significado de estas posiciones distara mucho de ser uniforme. Las características predominantes de los estratos bajos se habían modificado debido a la urbanización, al aumento de la movilidad espacial, a la desintegración parcial de las estructuras tradicionales del poder rural y al acceso a los medios de información. Pero como ya se ha dicho, no sólo no disminuía radicalmente la polarización entre 
tales estratos y los plenamente modernizados, sino que incluso sucedía lo contrario.

En el ámbito empresarial, los hechos de interés fueron las vinculaciones que se establecieron entre las distintas actividades económicas: por una parte, en muchos países se formó un empresariado rural moderno que incursionó en la exportación y en la agroindustria; pero, además, en el conjunto del sector empresarial se establecieron estrechas relaciones entre los sectores financiero, financiero-comercial e industrial. La vinculación con empresas externas siguió aumentando, tanto por el lado de la asociación directa como por el uso de patentes, marcas y tecnologías. Es interesante comprobar en ellas la existencia de ideologías en boga en los centros mundiales que reforzaron su identidad como grupo y el sentimiento de que desempeñaban un papel clave en la sociedad.

A la vez se observaba que los cuadros técnicos y científicos empezaban a asumir como marco de referencia intelectual el de sus pares en los países desarrollados, y se vinculaban a las orientaciones propias de dichos países en cuanto a eficiencia, especialización funcional, demandas de organización racional, concepción del Estado y otras. Muchos de los profesionales se vincularon a las empresas en funciones directivas, y adoptaron ideologías, estilos de vida y expectativas de consumo de tipo internacional; en cambio, la tecnocracia y la burocracia profesional vinculada al aparato del Estado en muchos casos vio que se deterioraban sus ingresos y sus condiciones de trabajo.

Los sectores medios asalariados siguieron constituidos por una heterogeneidad de grupos vinculados a funciones administrativas del Estado o a sus servicios sociales, como los de salud y educación, pero también adquirieron gran significación los asalariados vinculados al desarrollo de los servicios privados, financieros o de otro tipo, y a la expansión de los servicios comerciales que satisfacían un consumo urbano creciente. Estos sectores medios asalariados se vieron fuertemente afectados en los períodos de crisis — particularmente su sector pasivo-y su situación de inseguridad ha sido por lo regular permanente. Ha habido una 
apreciable distancia entre la realidad y sus aspiraciones de consumo moderno, y en varios casos se han detenido los procesos de movilidad ascendente que los favorecieron en el pasado.

Los estudios de la CEPAL mostraron que, sobre todo en los años ochenta, los trabajadores de las industrias y los ocupados en actividades afines experimentaron conmociones más graves aún que las que afectaron a los asalariados de los estratos medios; sufrieron reducciones radicales de sus salarios reales y el número de obreros industriales en algunos países mermó como proporción de una fuerza de trabajo urbana en constante crecimiento; además, en muchos países disminuyó la capacidad de los sindicatos para proteger los intereses de sus afiliados y fueron muy escasas sus posibilidades de intervenir en la política nacional.

Se comprobó asimismo que había habido una diversificación del campesinado, debido a los cambios en la estructura agraria y al surgimiento de formas diferentes a la tradicional relación de latifundio y minifundio. Se formaron empresas agrícolas que dieron origen a importantes grupos de asalariados, aunque en muchos casos sólo como trabajadores temporales; los minifundistas y colonos, por cierto, no han perdido significación. El surgimiento de empresas agrícolas y de un número considerable de asalariados supone un cambio en el tipo de relación del campesino con la tierra, el tipo de reivindicaciones y la consiguiente movilización. Por otra parte, el surgimiento de la agrodustria modificó también las actividades de los medianos y pequeños propietarios, que en muchos casos pasaron a relacionarse con ella; pero no ha perdido significación la "economía campesina" ligada a este tipo de propietarios, en la cual ha seguido predominando la estrategia de supervivencia del grupo familiar.

Por cierto, la marginalidad y la pobreza constituyeron temas de preocupación centrales de la CEPAL, y aunque ambas dimensiones no son estrictamente homologables, tampoco es posible desestimar sus relaciones. Los estudios de la CEPAL se orientaron al diagnóstico de estas situaciones y a establecer la relación entre ellas y las características del modelo vigente de desarrollo; se evaluó el impacto de las políticas sociales con relación a estos 
temas y se perfilaron estrategias para enfrentarlos. No obstante, el hecho de mayor interés fue haber mostrado que la pobreza y la marginalidad aparecen estrechamente asociadas al carácter excluyente y concentrador del estilo de desarrollo vigente, de modo que es necesario actuar sobre esos rasgos estructurales si se pretende superarlas.

Por último, con la intención de dar cuenta de la nueva situación social, la CEPAL abordó el análisis de dos categorías de extraordinaria significación: las mujeres y los jóvenes. Respecto de las mujeres, se mostró que los cambios no sólo incidían en el ámbito económico y social, sino que también afectaban los comportamientos culturales. La incorporación femenina a la actividad económica aumentaba con mucha rapidez, a tasas más altas que la masculina, aunque eso no significaba, por cierto, que participaran en la misma proporción, pues dicha actividad sigue favoreciendo a los hombres. Las mujeres se incorporaron preferentemente al sector de servicios, en muchos casos al de los llamados "servicios personales”, pero en la mayoría de los países las empleadas de oficina constituyeron el segundo rubro en importancia numérica.

El aumento de la participación femenina en la fuerza de trabajo apareció relacionado con la urbanización, la modernización, el proceso educativo y los cambios de percepción frente al trabajo. Del mismo modo, quedó en evidencia el creciente acceso de las mujeres a la educación, aunque la mayor igualdad de oportunidades se produjo en los niveles altos, manteniéndose discriminaciones en los grupos de condición social menos favorecida. Respecto a la participación social de la mujer, se comprobó que ésta es baja cuando se consideran mecanismos tradicionales de participación, como son los partidos políticos, los sindicatos u otras formas comunes de organización, pero la imagen varía si se consideran movimientos de carácter social vinculados a demandas ciudadanas específicas, como vivienda, educación, salud y mejores condiciones de vida. Es un hecho, por ejemplo, que entre los sectores populares muchas de estas reivindicaciones aparecían organizadas y lideradas por mujeres. Los cambios señalados incidieron en una modificación de la familia tradicional con una 
redistribución de los papeles en su interior; por lo demás, en los cambios de la estructura y el papel de la familia influyeron fuertemente la masificación educativa y el impacto de los medios de comunicación, que compitieron con la función socializadora de la familia.

El mundo de los jóvenes preocupó a la CEPAL por variadas razones. Por una parte, el peso numérico de ese tramo de edad, entre los 15 y 24 años, era en la región uno de los más elevados del mundo (alrededor del 20\% de la población) y de hecho un tercio de la fuerza de trabajo tenía menos de 75 años. Sin embargo, en los momentos de crisis, como en los años ochenta, la incorporación al empleo de los jóvenes era muy lenta y en algunos países ellos constituían casi la mitad del total de desempleados. De modo que existía plena conciencia de su precaria situación, pero además había una fuerte preocupación por su futuro. Se había aceptado como un hecho evidente por sí mismo que la expansión del sistema educacional facilitaría la incorporación positiva de los jóvenes al mundo del trabajo; pero empezaba a hacerse evidente la subutilización de capacidades de los más educados. Si bien los jóvenes aparecían como un sector social mucho más instruido que las generaciones adultas, sus oportunidades ocupacionales en un mundo de trabajo de reglas cambiantes y crecimiento lento no respondían a las expectativas generales.

Otro hecho relevante era la comprobación de la heterogeneidad de la juventud de la región como sector social. Los pertenecientes a familias en condiciones de pobreza tendían a quedar encerrados en circuitos de marginación. En los otros, paradójicamente, la propia organización y el carácter del sistema educativo se encargaba de acentuar la diferenciación, por la calidad dispar de la educación que impartían —independientemente de la equivalencia formal- los diversos tipos de establecimientos educacionales. Así, eran notorias esas diferencias entre establecimientos urbanos y rurales, o entre establecimientos privados y fiscales; también era visible la emergencia de sistemas universitarios que iban desde centros de excelencia hasta instituciones de mera acreditación, donde los conocimientos transmitidos no eran muy superiores a 
los de un colegio secundario. Por último, preocupaba también la construcción de identidad por los jóvenes, tema que aparecía como un serio desafío cultural, sobre todo si se tenía en cuenta tanto la proliferación de modelos exógenos que propagaban los medios de comunicación como los estilos de vida consumista.

\section{LA BÚSQUEDA DE UNA NUEVA PROPUESTA}

Ha sido casi un lugar común definir los años ochenta como "la década perdida". Si esto fue una realidad desde el punto de vista de los bajos rendimientos económicos y del deterioro de las condiciones sociales, no es menos cierto que ese momento de crisis fue un punto de inflexión en la modalidad de desarrollo hasta entonces vigente. El dinamismo anterior, impulsado por un sector exportador que dependía de productos primarios, una industrialización que se apoyaba principalmente en la demanda interna y una inversión que era fundamentalmente pública, tendía a agotarse y a ser reemplazado por nuevas modalidades. De suma importancia ha sido el cambio ideológico, en especial en lo que se refiere, desde el punto de vista del desarrollo, a la concepción del Estado. Éste pasó a concebirse como subsidiario, lo que quiere decir que el sector público debía desempeñar sólo aquellas actividades esenciales que el sector privado no podía o no quería realizar. Esta nueva ideología empezó a tomar cuerpo en casi todos los gobiernos de la región.

Por otra parte, en la mayoría de los países de la región comenzaron a surgir ciertos sectores caracterizados por una mayor modernidad productiva, muchos de ellos dedicados a la exportación de bienes no tradicionales, aunque también se produjeron importantes modernizaciones en los sectores financiero, de servicios y comercial. No obstante, el patrón distributivo del desarrollo latinoamericano no se modificó, y en varios casos incluso adquirió características más regresivas que antes. Y la estructura productiva, vinculada a un mercado interno concentrado en el cual la distribución del ingreso es muy desigual, ha reproducido las desigualdades. 
Actualmente se tiende a poner el acento en el crecimiento del sector más moderno, confiando en que generará una capacidad de arrastre suficiente para incorporar a los sectores y grupos sociales rezagados. La idea de apoyarse en los sectores más modernos se refuerza al considerar que se está asistiendo a una profunda revolución tecnológica de carácter mundial, que dejará irremisiblemente en la obsolescencia los modos anteriores de producción. Por lo demás, se ha pensado que el incremento de las exportaciones industriales puede contribuir a resolver el permanente desequilibrio externo de la mayoría de las economías de la región.

No obstante, en las nuevas modalidades de desarrollo que empiezan a perfilarse sigue siendo un punto de debate el tipo de relaciones que se constituyen entre el desarrollo económico - entendido como la expansión del producto y del ingreso por habitante y la distribución de ellos-y el desarrollo social, concebido como el mejoramiento de las condiciones de vida de la población.

La CEPAL, por supuesto, no ha estado ajena al debate y ha manifestado una especial preocupación por los aspectos sociales del desarrollo, sobre todo al tener en cuenta el alto costo social de la crisis de los años ochenta (desempleo y subempleo elevados, caída de los salarios reales, aumento de la pobreza y profundo deterioro de los servicios sociales). En la búsqueda de los factores que afectan al desarrollo social no se ha limitado al análisis de la eficiencia de los servicios sociales. Aunque importantes, no están en ellos las causas de la desigualdad social global. Como lo han puesto de manifiesto innumerables estudios cepalinos, los problemas reales se encuentran en las características de la relación recíproca entre las estructuras económicas, sociales y políticas.

La CEPAL ha avanzado una propuesta que se ha difundido con el nombre de "transformación productiva con equidad". En ella, las dimensiones sociales no aparecen sólo como un agregado: forman parte sustantiva de la propuesta. Entre esas dimensiones ocupa un lugar destacado el problema de la educación, dada la importancia preponderante que la actual revolución tecnológica confiere a la producción y utilización de conocimientos en el proceso de innovación. Por otra parte, las nuevas técnicas (la 
informática, las de comunicación) también tienen un innegable efecto sobre el proceso mismo de producción y difusión de conocimientos. La CEPAL, a través de sus propios estudios y los de otros organismos de las Naciones Unidas (principalmente la UNESCO) y también de estudios nacionales, ha tratado de mostrar los desafíos que se enfrentan en la región para lograr una adecuada articulación entre educación, desarrollo y equidad.

La expansión del sistema educativo después de la Segunda Guerra Mundial, y en especial en las tres últimas décadas, es innegable; no obstante, y sobre todo en el último tiempo, la mayor expansión se realizó en desmedro de la calidad de la enseñanza media impartida, y el uso de las capacidades del sistema fue poco eficiente. Esto fue particularmente notorio en la educación básica, que empezó a actuar como lastre de los demás niveles educativos, y a mostrar elevadas tasas de ingreso tardío, repetición, deserción temporal y deserción definitiva prematura. Con la expansión educativa se amplió también el acceso a la educación media, y ésta, que había sido concebida inicialmente como preparatoria del ingreso a la educación superior, se constituyó en un nivel masivo, tal como se suponía antes que debía ser la educación básica; no obstante, sus contenidos no se modificaron, y gran parte de quienes egresan de ella no salen preparados para incorporarse a la vida productiva. La propia calidad de la educación superior también está en tela de juicio, y pocas universidades cuentan con adecuados cuerpos estables de profesores e investigadores de jornada completa; proliferan los docentes de jornada parcial y los investigadores que llevan a cabo proyectos no siempre vinculados a las políticas universitarias de investigación.

Pero además los análisis ponen de manifiesto que el sistema educacional presenta una fuerte segmentación, que agrava la inequidad social existente. El bajo rendimiento, la deserción y la repetición se concentran en general en los sectores marginales urbanos y en el sector rural. Por lo demás, ha habido un fuerte deterioro de la capacidad de acción del Estado en el ámbito educacional, lo que provoca una crónica escasez de recursos materiales y humanos. Para quienes no tienen otra opción que recurrir a la 
educación estatal básica o media, ésta muchas veces toman más bien un carácter simbólico. Los grupos más privilegiados tienden a crear un circuito académico de mayor calidad, al que se ingresa de modo temprano a través de establecimientos preescolares o primarios de naturaleza privada y selectiva. De tal modo, una parte del sector privado se diferencia del sector fiscal y tiende a configurar circuitos escolares segmentados que se caracterizan por sus diferencias en el nivel de ingresos.

Como elemento importante, la propuesta de la CEPAL apunta a un tipo de desarrollo que fomente la cohesión social y consolide los procesos políticos democráticos en la región. La especial preocupación de la CEPAL por el problema de la participación ciudadana lleva al tema de las formas políticas que esta opción implica y el modo en que asume la democracia.

Democracia y participación adquieren, en la nueva propuesta de la CEPAL, el carácter de temas ineludibles de la dinámica de transformación. En tal sentido, el análisis sociológico busca comprender mejor las estructuras ideológicas vigentes y sus posibles orientaciones, el papel de las organizaciones y actitudes culturales, así como la función de los medios de comunicación. Y tiene particular relevancia la comprensión de la nueva relación que la sociedad establece con el Estado, puesto que una nueva relación Estado-sociedad es un elemento clave para alcanzar la sociedad más equitativa que la propuesta de la CEPAL postula.

\section{BIBLIOGRAFÍA}

Altimir, O., "La pobreza en América Latina: un examen de conceptos y datos", en Revista de la CEPAL, No 13, E/CEPAL G. 1.145, Santiago de Chile, abril de 1981.

, "Desarrollo, crisis y equidad", en Revista de la CEPAL, $\mathrm{N}^{\circ}$ 40, LC/G. 1.613-P, Santiago de Chile, abril de 1990.

Arriagada, I., "La participación desigual de la mujer en el mundo del trabajo", en Revista de la CEPAL, No 40, LC/G. 1.613-P, Santiago de Chile, abril de 1990. 
Baño, R., "Estructura socioeconómica y comportamiento colectivo", en Revista de la CEPAL, N 50, LC/G. 1.767-P, Santiago de Chile, agosto de 1993.

Brignol, R. y J. Crispi, "El campesinado en América Latina: una aproximación teórica”, en Revista de la CEPAL, N 16, E/CEPAL/G. 1.195, Santiago de Chile, abril de 1982.

Cardoso, F.H. y E. Faletto, Dependencia y desarrollo en América Latina: ensayo de interpretación sociológica, México, Siglo XXI, 1988.

Cardoso, F.H. y F. Weffort, "Introducción”, en América Latina: ensayos de interpretación sociológico-política, Santiago de Chile, Editorial Universitaria, 1970.

Cardoso, F.H., "La originalidad de la copia: la CEPAL y la idea del desarrollo", en Revista de la CEPAL, No 4, Santiago de Chile, publicación de las Naciones Unidas, Nº de venta S.77.II. G.5, 1977. CEPAL, El desarrollo social de América Latina en la posguerra, Buenos Aires, Solar/Hachette, 1963.

, La juventud en América Latina y el Caribe, LC/G. 1.345, Santiago de Chile, abril de 1985.

, Transformación productiva con equidad, LC/G.1.601-P, Santiago de Chile, publicación de las Naciones Unidas, $\mathrm{N}^{\circ}$ de venta S.90. II. G.6, 1990.

, Equidad y transformación productiva: un enfoque integrado.

LC/L. 668, Santiago de Chile, 6 de enero de 1992.

, Educación y conocimiento: eje de la transformación productiva con equidad, LC/G. 702/Rev. 2-P, Santiago de Chile, 1992.

Cibotti, R. y O. Bardeci, "Un enfoque crítico de la planificación en América Latina”, en Transformación y desarrollo: la gran tarea de América Latina, vol. 2, México, FCE, 1972.

Durston, J., "Clase y cultura en la transformación del campesinado", en Revista de la CEPAL, No 16, E/CEPAL/G. 1.195, Santiago de Chile, abril de 1982.

Fajnzylber, F., "Educación y transformación productiva con equidad", en Revista de la CEPAL, No 47. LC/G. 1.739-P, Santiago de Chile, agosto de 1992. 
Faletto, E., "La especificidad del Estado en América Latina", en Revista de la CEPAL, No 38, LC/G. 1.570-P, Santiago de Chile, agosto de 1989.

, "Formación histórica de la estratificación social en América Latina”, en Revista de la CEPAL, N 50, LC/G. 1.767-P, Santiago de Chile, agosto de 1993.

Filgueira, C. y C. Geneletti, "Estratificación y movilidad ocupacional en América Latina", en Cuadernos de la CEPAL, N³9, Santiago de Chile, 1981.

Filgueira, C., "Educar o no educar: ¿es éste el dilema?”, en Revista de la CEPAL, N² 21, E/CEPAL/G. 1.266, Santiago de Chile, diciembre de 1983 .

Franco, R., Tipología de América Latina: ensayo de medición de las discontinuidades sociales, Cuadernos del ILPES, Serie II: Anticipos de investigación, $\mathrm{N}^{\circ} 17$, Santiago de Chile, Instituto Latinoamericano y del Caribe de Planificación Económica y Social (ILPES), 1973.

, (comp.), Pobreza, necesidades básicas y desarrollo, Santiago de Chile, CEPAL/ILPES/Fondo de las Naciones Unidas para la Infancia (UNICEF), 1982.

Franco, R. y E. Cohen, "Racionalizando la política social: evaluación y viabilidad", en Revista de la CEPAL, N 47, LC/G. 1.739-P, Santiago de Chile, agosto de 1992.

Gerstenfeld, P. y R. Katzman, “Áreas duras y áreas blandas en el desarrollo social”, en Revista de la CEPAL, N 41, LC/G. 1.631-P, Santiago de Chile, agosto de 1990.

Graciarena, J., "Poder y estilos de desarrollo: una perspectiva heterodoxa", en Revista de la CEPAL, N ${ }^{\circ}$, Santiago de Chile, publicación de las Naciones Unidas. $\mathrm{N}^{\mathrm{o}}$ de venta S.76.II. G.2, 1976.

, "Tipos de concentración del ingreso y estilos políticos en América Latina”, en Revista de la CEPAL, $\mathrm{N}^{\circ} 2$, Santiago de Chile, publicación de las Naciones Unidas, $\mathrm{N}^{\mathrm{o}}$ de venta S.77.II. G.2, 1976.

, "La estrategia de las necesidades básicas como alternativa: sus posibilidades en el contexto latinoamericano", en Revista de la CEPAL, No 8, E/CEPAL/1089, Santiago de Chile, agosto de 1979. 
Graciarena, J. y R. Franco, Formaciones sociales y estructuras de poder en América Latina, Madrid, Centro de Investigaciones Sociológicas, 1981.

Gurrieri, A., "Vigencia del Estado planificador en la crisis actual", en Revista de la CEPAL, No 31, LC/G. 1.452, Santiago de Chile, abril de 1987.

Gurrieri, A, y E. Torres-Rivas (comp.), Los años noventa: ¿desarrollo con equidad?, Santiago de Chile, Facultad Latinoamericana de Ciencias Sociales (FLACSO)/CEPAL, 1990.

Kaztman, R., "Las transformaciones sectoriales del empleo en América Latina", en Revista de la CEPAL, N² 24. LC/G.1.324, Santiago de Chile, diciembre de 1984.

Krawczyk, M., "La creciente presencia de la mujer en el desarrollo", en Revista de la CEPAL, No 40, LC/G. 1.613-P, Santiago de Chile, abril de 1990.

, "Mujeres en la región: los grandes cambios", en Revista de la CEPAL, No 49, LC/G. 1.757-P, Santiago de Chile, abril de 1993.

Medina Echavarría, J., Aspectos sociales del desarrollo económico, Santiago de Chile, Editorial Andrés Bello, 1959.

"Un modelo teórico de desarrollo aplicable a América Latina", en Aspectos sociales del desarrollo económico en América Latina, Bruselas, UNESCO, 1962.

, Consideraciones sociológicas sobre el desarrollo económico en América Latina, Buenos Aires, Solar/Hachette, 1964. , Discurso sobre política y planeación, México, Siglo XXI, 1972.

Aspectos sociales del desarrollo económico, Santiago de Chile, CEPAL, 1973.

Pensamiento iberoamericano: cambios en la estructura social, $\mathrm{N}^{\circ} 6$, Madrid, julio-diciembre de 1984.

Pinto, A., "Notas sobre los estilos de desarrollo en América Latina", en Revista de la CEPAL, $\mathrm{N}^{\circ} 1$, Santiago de Chile, publicación de las Naciones Unidas, $N^{\circ}$ de venta S.76.II. G 2, 1970.

Quijano, A., "Dependencia, cambio social y urbanización en América Latina”, en Revista Mexicana de Sociología, vol. 30, N 3 , México, Universidad Autónoma de México (UNAM), 1968. 
Rama, G., "La educación latinoamericana: exclusión o participación”, en Revista de la CEPAL, N²1, E/CEPAL/G. 1.266, Santiago de Chile, diciembre de 1983.

, "La juventud latinoamericana ante el desarrollo y la crisis", en Revista de la CEPAL, N²9, LC/G. 1.427, Santiago de Chile, agosto de 1986.

Rama, G. y E. Faletto, "Sociedades dependientes y crisis en América Latina: los desafíos de la transformación político-social”, en Revista de La CEPAL, N 25, LC/G. 1.338, Santiago de Chile, abril de 1985.

Rosenbluth, G., "Informalidad y pobreza en América Latina”, en Revista de la CEPAL, N 52, LC/G. 1.824-P, Santiago de Chile, abril de 1994.

Solari, A., R. Franco y J. Jutkowitz, Teoría, acción social y desarrollo en América Latina, México, Siglo XXI Editores, 1976.

Solari, A., "Desarrollo y política educacional en América Latina", en Revista de la CEPAL, N ${ }^{\circ}$ 3, Santiago de Chile, publicación de las Naciones Unidas, $\mathrm{N}^{\mathrm{o}}$ de venta S.77.II-G.3, 1977.

Sonntag, Heinz, Duda, certeza: la evolución de las ciencias sociales de América Latina, Caracas, UNESCO/Nueva Sociedad, 1988.

Weffort, Francisco, Clases populares y desarrollo social, Santiago de Chile, ILPES, 1968.

Wolfe, M., El desarrollo esquivo: exploraciones en la política social y la realidad sociopolítica, México, FCE, 1976.

, "Los actores sociales y las opciones de desarrollo", en Revista de la CEPAL, N 35, LC/G. 1527-P, Santiago de Chile, agosto de 1988.

, "Las estructuras sociales y la democracia en los años noventa”, en Revista de la CEPAL, No 40, LC/G. 1.613-P, Santiago de Chile, abril de 1990. 